Published in final edited form as:

Expert Opin Pharmacother. 2013 August ; 14(12): 1695-1703. doi:10.1517/14656566.2013.812632.

\title{
Dapagliflozin for the treatment of type 2 diabetes
}

\author{
Muhammad A Abdul-Ghani, MD, $\mathrm{PhD}^{\dagger}$ and Ralph A DeFronzo, MD \\ University of Texas Health Science Center, Division of Diabetes, San Antonio, TX, USA
}

\begin{abstract}
Introduction-Despite the availability of many antihypreglycemic agents, many patients with type 2 diabetes (T2DM) fail to achieve the glycemic treatment goal, primarily due to progressive beta-cell dysfunction, and increased risk of hypoglycemia.
\end{abstract}

Areas covered-The aim of the present article is to review the efficacy and safety of dapagliflozin, a novel antihyperglycemic drug that lowers the plasma glucose concentration by the inhibition of renal sodium-glucose cotransport, in lowering the plasma glucose concentration and the HbA1c in T2DM patents. This review summarizes the published data about the mechanism of action and clinical efficacy of dapagliflozin in lowering the HbA1c in patients with T2DM. It also discusses additional non-glycemic benefits of dapagliflozin and the safety profile of the drug.

Expert opinion-Dapagliflozin is effective in lowering the plasma glucose concentration in patients with T2DM with a good safety profile. Because of its unique mechanism of action, dapagliflozin can be utilized in combination with all other antihyperglycemic agents and at all stages of the disease.

\section{Keywords}

dapagliflozin; kidney; SGLT2 inhibition; sodium-glucose cotransport; type 2 diabetes

\section{Background}

Type 2 diabetes (T2DM) affects $\sim 25$ million individuals in the USA and $>300$ million people worldwide [1]. Hyperglycemia is the principal risk factor for diabetic microvascular complications, and it also plays an important role in the pathogenesis of insulin resistance and beta-cell failure, that is, glucotoxicity [2,3]. Thus, improved glycemic control in subjects with diabetes would be anticipated to reduce the risk of microvascular complications and ameliorate the metabolic abnormalities that contribute to the development and progression of hyperglycemia. Despite the irrefutable evidence implicating hyperglycemia in the development of microvascular complications, about one-half of people with diabetes in the USA and worldwide fail to achieve the recommended target of glycemic control, $\mathrm{HbA} 1 \mathrm{c}<$ $6.5-7 \%$, with currently available antidiabetic medications [4,5].

\footnotetext{
${ }^{\dagger}$ Author for correspondence: University of Texas Health Science Center, Division of Diabetes, 7703 Floyd Curl Dr, MSC7886, San Antonio, TX 78229, USA, Tel: +1 210567 6691; Fax: +1 210567 6554; abdulghani@uthscsa.edu.

Declaration of interest

MA Abdul-Ghani has no conflicts of interest.
} 
Progressive beta-cell failure, weight gain and hypoglycemia represent major obstacles when attempting to achieve optimal glycemic control (HbA1c $\leq 6.5-7.0 \%)$ in patients with T2DM [2]. Although conventional antidiabetic therapies, for example, metformin, sulfonylurea and insulin, are effective in lowering the plasma glucose concentration, they do not halt the progressive beta-cell dysfunction, and, the HbA1c rises progressively after an initial decline in subjects receiving these therapies. Moreover, both insulin and sulfonylureas are associated with an increased rate of hypoglycemia which often precludes the achievement of the target HbA1c goal. Moreover, insulin and sulfonylurea therapy often are associated with weight gain which causes insulin resistance, thereby antagonizing the glucose-lowering efficacy of these antidiabetic agents. Therefore, novel therapies that effectively lower the plasma glucose concentration and produce durable glycemic control, while avoiding hypoglycemia and weight gain, are needed for the management of T2DM patients. Dapagliflozin (Box 1) is a member of a new class of antidiabetic agents, the renal sodium-glucose cotransporter 2 (SGLT2) inhibitors, which have been developed for the treatment of T2DM [6]. In this review, the authors will summarize the available data concerning the mechanism of action, efficacy and safety of dapagliflozin.

\section{Renal handling of glucose}

In normal healthy individuals with a fasting plasma glucose concentration of $80-90 \mathrm{mg} / \mathrm{dl}$ and a glomerular filtration rate (GFR) of 180 1/day, the kidney filters $\sim 162 \mathrm{~g}$ of glucose. Virtually, all of this glucose is reabsorbed in the proximal tubule and no glucose is excreted in the urine. The maximum glucose transport capacity $(\mathrm{Tm})$ of the proximal tubule is, on average, $\sim 375 \mathrm{mg} / \mathrm{min}$. Two sodium-glucose cotransporters, SGLT1 and SGLT2, which are located in the $\mathrm{S} 3$ and $\mathrm{S} 1$ segments of the proximal tubule, respectively, are responsible for reabsorption of virtually $100 \%$ of the filtered glucose load [7].

\subsection{Renal sodium-glucose reuptake}

Renal glucose reabsorption by SGLT1 and SGLT2 is coupled to sodium reabsorption. The electrochemical gradient generated by active sodium transport provides the energy required for glucose transport (Figure 1). The SGLT2 transporter is expressed exclusively in the kidney [7]. It has a low affinity and high capacity for glucose transport, and is responsible for the reabsorption of $\sim 80-90 \%$ of the filtered glucose. The remaining $10-20 \%$ of filtered glucose is reabsorbed by the SGLT1 transporter [7]. SGLT1 is expressed in the kidney and gut, transports both glucose and galactose, and is responsible for the majority of glucose and galactose uptake in the gut. In normal individuals, the filtered glucose load is less than the maximal glucose transport capacity ( $\mathrm{Tm} \sim 375 \mathrm{mg} / \mathrm{min}$ ), resulting in complete absorption of all of the filtered glucose. If the filtered glucose load exceeds $375 \mathrm{mg} / \mathrm{min}$, as often occurs in poorly controlled diabetic individuals, the Tm is exceeded and glucose in excess of the Tm is excreted in the urine [6]. The plasma glucose concentration at which the filtered glucose load reaches the $\mathrm{Tm}$ is called the threshold and in normal glucose tolerant (NGT) individuals the threshold is $\sim 180 \mathrm{mg} / \mathrm{dl}$. At plasma glucose levels above the threshold, the glucose excretion rate increases linearly and parallels the filtered glucose load. The reabsorption and excretion curves display a non-linear transition as the Tm for glucose is approached. This 'rounding' of the curves is termed splay (Figure 2) and has been 
explained by heterogeneity in the Tm of individual nephrons and/or glomerulotubular imbalance [6].

\subsection{Renal glucose handling in T2DM}

Previous studies have reported an increase in the maximum glucose reabsorptive capacity $\left(\mathrm{Tm}_{\mathrm{G}}\right)$ in subjects withT2DM and in animal models of diabetes [8,9]. Reduction of the elevated plasma glucose levels led to decrease in the $\mathrm{Tm}_{\mathrm{G}}$. The increase in $\mathrm{Tm}$ in response to hyperglycemia results in enhanced renal glucose reabsorption compared with normal glucose tolerance individuals, implicating the kidney as an active participant in the pathogenesis of hyperglycemia in T2DM subjects.

\section{Inhibition of renal glucose uptake by dapagliflozin}

Dapagliflozin is a potent inhibitor of SGLT2 with an $\mathrm{IC}_{50}$ value of $1.1 \mathrm{nM}$ [10]. The $\mathrm{IC}_{50}$ ratio for SGLT2 inhibition to SGLT1 inhibition is 1200 . Thus, dapagliflozin is a highly selective and potent inhibitor of SGLT2. Single dose administration of dapagliflozin produces a dose-dependent glucosuria in NGT individuals that lasts for $>24 \mathrm{~h} \mathrm{[11].} \mathrm{The}$ maximal rate of glucosuria is $\sim 3 \mathrm{~g} / \mathrm{h}$ and is achieved with a single dapagliflozin dose of $20-$ $25 \mathrm{mg}$. With multiple dosing, dapagliflozin causes a dose-dependent glucosuria with a maximal glucose excretion rate achieved with the $20 \mathrm{mg}$ dose. The cumulative amount of glucose excreted per 24 in NGT individuals was 58 and $49 \mathrm{~g}$ at 1 and 14 days, respectively, following initiation of dapagliflozin treatment with $20 \mathrm{mg}$ daily dose [11]. This amount of glucosuria represents $<50 \%$ of the filtered glucose load in NGT individual. The failure to observe greater inhibition of the filtered glucose load can be explained by the fact that under complete SGLT2 inhibition, the fraction of filtered glucose reabsorbed by SGLT1 is significantly enhanced $[12,13]$. Thus, only the amount of filtered glucose that escapes SGLT1 is excreted in the urine.

\section{Mechanism of glucosuria}

Glucosuria can be promoted by lowering the Tm, increasing the glucose splay, or reducing the threshold. Dapagliflozin produces a marked decrease in both the Tm and splay in diabetic and non-diabetic individuals [14]. However, the decrease in Tm caused by dapagliflozin is not sufficient to explain the marked glucosuria observed in NGT individuals with a fasting plasma glucose concentration in the $80-90 \mathrm{mg} / \mathrm{dl}$ range [14]. The major mechanism via which dapagliflozin induces glucosuria in NGT, as well as in diabetic individuals, is related to a marked reduction in the threshold (from 180 to $\sim 40 \mathrm{mg} / \mathrm{dl}$ ) at which plasma glucose starts to spill into the urine [14].

\section{Pharmacokinetics of dapagliflozin}

Dapagliflozin is rapidly absorbed after oral administration with bioavailability $>75 \%$ [11]. The maximum plasma dapagliflozin concentration $\left(\mathrm{C}_{\max }\right)$ is reached within $2 \mathrm{~h}$, and it is delayed by $30 \mathrm{~min}$ and decreased by $39 \%$ after a high fat meal. The effect of high-fat diet on dapagliflozin availability is unlikely to be clinically significant because the area under the plasma drug concentration is only slightly $(\sim 5 \%)$ affected by food [15]. Dapagliflozin has a 
long half-life (13.8 h), which makes it suitable for once-daily dosing [16]. More than $90 \%$ of plasma dapagliflozin is bound to plasma proteins, and only $2 \%$ of the parent drug is cleared by the kidney. Dapagliflozin is metabolized in the liver to an inactive metabolite (dapagliflozin 3-O-glucuronide), which represents the major metabolite. Hepatic metabolism also produces an active metabolite ( $O$-deethylated dapagliflozin). However, this active metabolite is not generated with dapagliflozin doses $<50 \mathrm{mg}$ [16]. No interaction with hepatic metabolized drugs, for example, diuretics, angiotensin receptor blockers (ARBs) or angiotensin-converting enzyme (ACE) inhibitors [12] or other antihyperglycemic agents [17] has been observed with dapagliflozin and clinical studies have not demonstrated any need for dose adjustment in subjects with mild to moderate hepatic impairment [16]. The drug pharmacokinetic properties are not affected by age, ethnicity, presence of T2DM or body mass index [16]. Of note, the drug's efficacy is reduced in diabetic individuals with impaired renal function (see discussion below).

\section{Clinical efficacy of dapagliflozin}

The efficacy of dapagliflozin in lowering the plasma glucose concentration and HbAlc in T2DM individuals has been examined in randomized, placebo-controlled, double-blind trials and against active comparators in drug-naïve individuals, in poorly controlled individuals on oral antidiabetic agent therapy and in insulin-treated diabetic individuals (Figure 3).

\subsection{Dapagliflozin in drug-naïve T2DM patients}

Multiple trials have examined the efficacy of dapagliflozin in lowering the HbA1c in drugnaïve T2DM individuals. In a 24-week multicenter study [18], 485 diabetic individuals were randomized to receive $2.5,5$ and $10 \mathrm{mg}$ dapagliflozin or placebo for 24 weeks. The placebosubtracted decrease in $\mathrm{HbA} 1 \mathrm{c}$ in dapagliflozin-treated subjects was $0.5-0.6 \%$ (from a baseline $\mathrm{HbA} 1 \mathrm{c}$ of $7.8-8.0 \%$ ). The decrease in HbA1c was slightly smaller with the $2.5 \mathrm{mg}$ $(0.48 \%)$ than with the 5 and $10 \mathrm{mg}$ doses $(0.6 \%)$. Dapagliflozin lowered the FPG by $\sim 25$ $\mathrm{mg} / \mathrm{dl}$ (from a baseline FPG of $160 \mathrm{mg} / \mathrm{dl}$ ). In a subgroup $(\mathrm{n}=78)$ of subjects with baseline $\mathrm{HbA1c}$ of $10.1-12.0 \%$, dapagliflozin ( 5 and $10 \mathrm{mg} /$ day) reduced the HbA1c by 2.88 and $2.66 \%$, respectively. In another 24-week study [19], dapagliflozin (doses $=1,2.5,5 \mathrm{mg}$ ) was examined in 282 drug-naïve T2DM individuals and compared with placebo. Dapagliflozin lowered the HbA1c by $0.7-0.8 \%$ from a baseline HbA1c $=7.8-8.1 \%$. The placebosubtracted decrease in $\mathrm{HbAlc}$ was slightly greater with the $5 \mathrm{mg}$ dose $(0.84 \%)$ compared with the other two lower doses ( 0.70 and $0.74 \%$ for 1 and $2.5 \mathrm{mg}$, respectively). The efficacy of dapagliflozin (1, 2.5, 5 and $10 \mathrm{mg} /$ dose vs placebo) was also examined in 279 Japanese diabetic individuals in a 12-week study [20]. The 1 and $2.5 \mathrm{mg}$ doses produced a similar decrease $(\sim 0.5 \%)$ in the $\mathrm{HbAl}$ c, which was smaller than the reduction $(0.74$ and $0.80 \%)$ produced by the 5 and $10 \mathrm{mg}$ doses.

Two other studies [21,22] have compared the efficacy of dapagliflozin versus metformin in lowering the HbA1c in drug-naïve diabetic individuals. In a 12-week study [21], dapagliflozin (doses $=2.5,5,10,20$ and $50 \mathrm{mg}$ ) was compared with metformin in 440 drugnaïve diabetic individuals. Dapagliflozin reduced the HbA1c by $0.7 \%$ (from a baseline $\mathrm{HbA1c}$ of $7.8-8.0 \%$ ) without any apparent dose-dependency, and the reduction in $\mathrm{HbA1c}$ 
was similar in magnitude to that observed with metformin $(-0.73 \%)$. The reduction in fasting and postprandial plasma glucose concentrations accounted approximately equally for the decline in HbA1c. Another 24-week study [22] compared the efficacy of 5 and $10 \mathrm{mg}$ of dapagliflozin alone or in combination with metformin in lowering the HbA1c in 1236 drugnaïve diabetic individuals. Both dapagliflozin doses $(5$ and $10 \mathrm{mg})$ produced a significant reduction in $\mathrm{HbAc1}$ (1.19 and $1.45 \%$, respectively) which was comparable with that produced by metformin $(1.40 \%)$. The high baseline HbAlc $(9.1 \%)$ in this study explains the greater decrease in HbA1c compared with previous studies. Importantly, both dapagliflozin doses produced an additive effect in lowering the HbA1c when combined with metformin and the decrease in metformin plus dapagliflozin was 2.05 and $1.98 \%$ for the 5 and $10 \mathrm{mg}$ doses, respectively. Fifty-two and $47 \%$ of participants, respectively, achieved an HbA1c goal of $<7.0 \%$ compared with only $35 \%$ of metformin-treated individuals.

\subsection{Dapagliflozin as add-on therapy}

The efficacy of dapagliflozin in lowering the plasma glucose concentration as add-on therapy has been examined in poorly controlled T2DM treated with metformin, sulfonylureas and pioglitazone. Dapagliflozin effectively reduced the HbA1c in poorly controlled diabetic individuals independent of the background therapy. In a 24-week study [23], 546 metformin-treated individuals with $\mathrm{HbA1c} 7-10 \%$ were randomized to receive $2.5,5$ and $10 \mathrm{mg}$ of dapagliflozin or placebo. The placebo-subtracted reduction in HbA1c at 24 weeks was $-0.37,-0.40$ and $-0.54 \%$, respectively. Dapagliflozin also produced a dosedependent decrease in FPG (12, 16 and $18 \mathrm{mg} / \mathrm{dl}$, respectively). Seventy-one percent of subjects in this study participated in a 102-week extension study and the decrease in HbA1c was maintained at 102 weeks [24]. As add-on therapy, dapagliflozin has been shown to have similar potency to sulfonylureas in lowering the HbA1c in poorly controlled metformintreated diabetic subjects. In a 52-week head-to-head study [25], 814 meformin-treated subjects $(\mathrm{HbA} 1 \mathrm{c}>6.5 \%)$ were randomized to receive dapagliflozin or glipizide. After an 8week lead in period, dapagliflozin and glipizide were up titrated over 18 weeks to 10 and 20 $\mathrm{mg}$, respectively. The decrease in HbA1c at 52 weeks was identical in both groups $(0.52 \%)$ [25], and the decrease in HbA1c in dapagliflozin-treated subjects was maintained at 102 weeks [26]. In a 24-week study [27], 597 sulfonylurea-treated subjects with poor glycemic control (HbA1c $7-10 \%$ ) were randomized to receive 2.5, 5 and $10 \mathrm{mg}$ of dapagliflozin or placebo. The placebo-subtracted decrease in HbA1c was $-0.44,-0.49$ and $-0.68 \%$, respectively. Dapagliflozin (5 and $10 \mathrm{mg}$ ) has also been examined in a 24-week study [28] in 420 poorly controlled (mean HbA1c $8.4 \%$ ), pioglitazone-treated T2DM subjects. The placebo-subtracted decrease in $\mathrm{HbA1c}$ was -0.40 and $-0.55 \%$, respectively, and the decrease in HbAlc was maintained at 48 weeks. Dapagliflozin equally reduced the fasting and the postprandial plasma glucose concentration by $\sim 25 \mathrm{mg} / \mathrm{dl}$.

\subsection{Dapagliflozin in insulin-treated subjects}

Because the mechanism of action of dapagliflozin is independent of insulin secretion and insulin action, the efficacy of dapagliflozin is independent of beta-cell function or diabetes duration. Thus, dapagliflozin is effective in reducing the HbA1c in insulin-deficient patients. In a pilot study, Wilding et al. [29] randomized 71 insulin-treated ( $\geq 50$ units/day) type 2 diabetic patients who also were receiving an insulin sensitizer (metformin and/or 
thiazolidinedione) as add-on therapy with dapagliflozin ( 5 and $10 \mathrm{mg} / \mathrm{day}$ ) or placebo. The insulin dose was reduced by $50 \%$ at the start of therapy, while the insulin sensitizer dose was unchanged. After 12 weeks, the placebo-subtracted decline in $\mathrm{HbA} 1 \mathrm{c}$ was 0.70 and $0.78 \%$, respectively ( $\mathrm{p}<0.01 \mathrm{vs}$ placebo) despite the $50 \%$ reduction in insulin dose. In a larger study $(\mathrm{n}=800)$ [30], the addition of dapagliflozin $(2.5,5$ and $10 \mathrm{mg} /$ day) to insulin-treated T2DM individuals (receiving $~ 70-80$ units/day for a mean of $\sim 6$ years) caused a dosedependent decrease in HbA1c $(-0.40,-0.49$ and $-0.57 \%$, respectively) compared with placebo over 24 weeks and the decrease in HbA1c was maintained at 48 weeks [30]. Further, the reduction in $\mathrm{HbAlc}$ was independent of the diabetes duration. To further examine the efficacy of dapagliflozin as a function of duration of diabetes, 151 subjects with new-onset diabetes ( $<1$ year) and 58 subjects with long-standing ( 11 years) T2DM were randomly assigned to 10 or $20 \mathrm{mg} /$ day of dapagliflozin for 12 weeks [31]. Although subjects with long-standing diabetes were in poor glycemic control (HbA1c 8.4\%) despite a large dose of insulin (> 50 units/day) plus metformin and a thiazolidinedione, dapagliflozin caused a comparable reduction in $\mathrm{HbA1c}$ in both groups.

\section{Dapagliflozin and renal function}

Studies with dapagliflozin have demonstrated that treatment with an SGLT2 inhibitor has no deleterious effect on renal function [32]. Moreover, because the majority of drug's clearance is by the liver, no dose adjustment is necessary in patients with renal impairment [32].

Because of its mechanism of action, the efficacy of dapagliflozin to reduce plasma glucose concentrations is highly dependent on the level of renal function. As the GFR declines, there is a decrease in the filtered glucose load and a progressive decrease in the glucose-lowering ability of the drug (Figure 4). In Phase III trials, subjects with a mild decrease in renal function (GFR $60-90 \mathrm{ml} / \mathrm{min} / 1.73 \mathrm{~m}^{2}$ ), $10 \mathrm{mg}$ dapagliflozin produced $0.54 \%$ decrease compared with $0.69 \%$ in subjects with GFR $>90 \mathrm{ml} / \mathrm{min} / 1.73 \mathrm{~m}^{2}$, while in subjects with GFR $<60 \mathrm{ml} / \mathrm{min} / 1.73 \mathrm{~m}^{2}$, the decrease in HbA1c was only $0.39 \%$ (Figure 4). In a study which specifically was designed to examine the impact of impaired renal failure [33] on the efficacy of dapagliflozin, the mean decrease in HbA1c in 252 subjects with a mean GFR $44.6 \mathrm{ml} / \mathrm{min} / 1.73 \mathrm{~m}^{2}$ was only $0.11 \%$, which was significantly smaller than that observed from the analysis of subjects in Phase III trials. A further analysis indicated that the small decrease in HbA1c in the latter study was driven by subjects with GFR $<45 \mathrm{ml} / \mathrm{min} / 1.73$ $\mathrm{m}^{2}$, in whom the placebo-subtracted decrease in HBA1c was $0.07 \%$. In summary, as the GFR decreases, the clinical efficacy of dapagliflozin diminishes and as GFR approaches $45 \mathrm{ml} / \mathrm{min} / 1.73 \mathrm{~m}^{2}$, the drug completely losses its efficacy. In subjects with estimated glomerular filtration rate (eGFR) $45-60 \mathrm{ml} / \mathrm{min} / 1.73 \mathrm{~m}^{2}$, dapagliflozin dose should be reduced to $5 \mathrm{mg} /$ day because of increased incidence of side effects.

\section{Non-glycemic benefits of dapagliflozin}

In addition to the beneficial effects related to improved glycemic control, dapagliflozin exerts additional non-glycemic benefits in T2DM patients that make the drug desirable as monotherapy and as combination therapy. 


\subsection{Weight loss}

Weight gain is a major problem with many currently available antidiabetic medications including sulfonylureas, thiazolidinediones and insulin. The urinary loss of $60-80 \mathrm{~g}$ of glucose per day equates to a negative energy balance of $240-320 \mathrm{cal} /$ day or $2-3$ pounds/ month if this caloric deficit is not offset by an increase in caloric intake. Consistent with this, weight loss was observed in diabetic subjects treated with dapagliflozin at all doses, when the drug was administered as monotherapy [18-22] or in combination with other antidiabetic therapies [23-30] and at all stages of the disease [31]. The weight loss primarily is due to a decrease in fat mass [34], ranges between 2 and $3 \mathrm{~kg}$ and plateaus approximately 1 year after starting the drug.

\subsection{Blood pressure}

A consistent finding in all dapagliflozin studies has been a reduction in systolic/diastolic blood pressure of $4-5 / 2-3 \mathrm{~mm} \mathrm{Hg}$ [18-31]. Although this has been attributed to the mild fluid/sodium deficit that occurs during the first several days of dapagliflozin treatment, an alternative explanation is local inhibition of the renin-angiotensin system secondary to enhanced sodium delivery to the juxtaglomerulosa apparatus [35,36]. A recent micropuncture study [37] in experimental animals demonstrated that acute inhibition of SGLT2 causes two- to threefold increase in single nephron sodium excretion. However, the increased sodium excretion waned after 2 weeks, suggesting an important role of tubuloglomerular feedback in the long-term balance of fluid and salt. Because uric acid and sodium reabsorption in the proximal tubule are coupled, it is not surprising that a decrease in serum uric acid concentration consistently has been observed in diabetic patients treated with dapagliflozin [36].

\subsection{Diabetic nephropathy}

Hyperglycemia is the principal risk factor for diabetic microvascular complications (retinopathy, nephropathy and neuropathy), and improved glycemic control - no matter how achieved - would be expected to reduce the risk of microvascular complications in patients with T2DM $[38,39]$. Enhanced sodium-glucose reabsorption in the proximal tubule has been shown to play an important role in the development of diabetic nephropathy [35,36]. In hyperglycemic diabetic individuals the increased filtered glucose load results in increased glucose and sodium reabsorption by the SGLT2 transporter in the proximal tubule [40,41], thereby reducing sodium delivery to the juxtaglomerular apparatus. This activates the local renin-angiotensin system resulting in elevated intraglomerular pressure and glomerular damage even though the GFR is increased initially [42]. The SGLT2 inhibitors block glucose/sodium reabsorption in the proximal tubule. This increases sodium delivery to the juxtaglomerular apparatus, leading to inhibition of renin and angiotensin secretion and a reduction in intraglomerular pressure and hyperfiltration. On a long-term basis, the reduction in intraglomerular pressure would be expected to preserve renal function. Consistent with this, hyperfiltration and increased kidney size can be reversed by 6 weeks of intensive insulin therapy that normalizes the plasma glucose concentration and reduces the filtered glucose load [43]. 


\section{Safety}

In general, dapagliflozin is well tolerated and no major adverse events have been reported with the drug. Because the number of subjects above age 70 years in Phase III trials was small, dapagliflozin should be used with caution in this age group.

\subsection{Effect on water and electrolyte balance}

Dapagliflozin causes a modest increase in urine volume and sodium excretion during the initial $2-3$ days of therapy. During $12-24$ weeks of treatment with dapagliflozin, a modest salt/water deficit which results in $200-400 \mathrm{ml}$ increase in urine volume without excessive losses of potassium or other electrolytes has been documented [11]. Consistent with the mild intravascular volume contraction, a small rise in hematocrit $(1-2 \%)$ and plasma urea nitrogen to creatinine ratio has been observed. Plasma electrolyte concentrations did not change following dapagliflozin [18-31].

\subsection{Hypoglycemia}

No significant increase in the incidence of hypoglycemia has been observed when dapagliflozin was given to drug-naïve diabetic patients or pioglitazone-treated diabetic patients. When dapagliflozin was added to sulfonylurea-treated subjects, there was a small non-significant, but detectable, increase in the incidence of minor hypoglycemic events [27]. Similarly, when added to insulin-treated subjects, there was a slight increase in the incidence of hypoglycemic events which required a modest decrease in insulin dose [29].

\subsection{Urogenital infections}

Dapagliflozin promotes glucosuria which could result in an increased incidence of urinary tract infections. However, patients with uncontrolled blood sugar already have significant glucosuria. It remains to be determined whether the additional glucosuria will promote bacterial growth. In one study in which mid-stream urine was collected, treatment with dapagliflozin did not increase the prevalence of urinary bacteriuria [44]. However, in Phase III trials, diabetic subjects who received dapagliflozin at doses of $5 \mathrm{mg}(\mathrm{n}=1145)$ and $10(\mathrm{n}$ $=1193) \mathrm{mg} /$ day manifested a small increase in the incidence of urinary tract infection [1831]. The incidence of vulvo-vaginitis and balanitis is increased approximately twofold with dapagliflozin therapy [18-31] and usually responds to local antifungal therapy.

\subsection{Renal function}

As previously discussed, dapagliflozin has no significant effect on renal function, as manifested by an increase in serum creatinine or development of albuminuria or tubular proteinuria in diabetic subjects with normal GFR [18-31]. Dapagliflozin had no effect on plasma calcium concentration or bone reabsorption markers [45].

\subsection{Risk of cancer}

In Phase III studies with dapagliflozin, an increased incidence of bladder and breast cancer was observed. However, the total number of cases was small, 9 versus 1 for each cancer with a 2:1 randomization scheme, dapagliflozin:placebo. This finding is surprising because 
neither breast nor bladder tissues expresses the SGLT2 transporter. Chronic increase in urinary glucose excretion potentially could affect the risk of bladder cancer. However, rigorous 2-year carcinogenic studies in animals failed to demonstrate any preneoplastic or neoplastic activity, and an increased incidence of bladder cancer has not been reported in subjects with familial renal glucosuria. Moreover, because breast and especially bladder cancer take many years to develop, whereas the exposure to dapagliflozin was short (generally $<1$ year), the significance of the increased incidence of these two tumors remain to be determined. Further, five of cases of bladder cancer had hematuria at time of entry into the study. Moreover, there could have been detection bias for bladder cancer due to the frequent testing for urinary tract infections, and this could have led to the discovery of microscopic hematuria. Because of this potential carcinogenic signal, the Food and Drug Administration (FDA) has asked for additional safety data regarding cancer risk with dapagliflozin before approval of the drug for clinical use. Nonetheless, the drug has received the approval by European Medicines Agency (EMA) and is now available for clinical use in Europe.

As with every novel therapeutic agent, long-term information about its safety will require follow-up post-marketing studies, and such studies have been proposed by the manufacturer.

\section{Expert opinion}

Dapagliflozin is an effective novel strategy to reduce the plasma glucose concentration in type 2 diabetic subjects. The drug's unique mechanism of action, which is independent of insulin sensitivity and $\beta$-cell function, allows the use of dapagliflozin in combination with all other antidiabetic agents and at all stages of the disease (Figure 3). Indeed, dapagliflozin was equally efficacious in reducing $\mathrm{HbAlc}$ in drug-naïve individuals, in combination with other oral antidiabetic agents and in insulin-treated T2DM [18-31].

All major organizations (American Diabetes Association (ADA)/European Association for the Study of Diabetes (EASD)/American Association of Clinical Endocrinologists (AACE)) recommend metformin as first-line therapy in individuals with new-onset diabetes. However, because metformin does not affect $\beta$-cell function, metformin-treated individuals experience a progressive increase in HbA1c after the first year of therapy. Dapagliflozin can provide an effective therapeutic option in metformin-failing diabetic individuals. An increase in the plasma glucagon-like peptide-1 (GLP-1) concentration has been observed in some clinical studies following initiation of dapagliflozin therapy. This observation makes the double combination of dapagliflozin plus a dipeptidyl peptidase IV (DPP-IV) inhibitor an attractive therapeutic option in metformin-failing individuals. In newly diagnosed T2DM individuals with a very high $\mathrm{HbA1c}$ (e.g., > 9.0\%), metformin alone will not lower the HbA1c to the treatment goal $(<7.0 \%)$. Because dapagliflozin plus metformin have an additive effect to lower the $\mathrm{HbA1c}$, and because dapagliflozin produces a profound decrease in HbA1c in patients with a high initial HbA1c (> 9.0\%), the combination of dapagliflozin plus metformin is an attractive therapeutic option in newly diagnosed diabetic individuals with a high starting HbA1c. Metformin cannot be started in about $10-20 \%$ of subjects with newonset diabetes due to either gastrointestinal (GI) side effects or due to renal failure. Thus, dapagliflozin, as monotherapy or in combination with a DPP-IV inhibitor, can provide an 
effective initial therapy in metformin-intolerant individuals. Because dapagliflozin is effective in lowering the $\mathrm{HbA} 1 \mathrm{c}$ at all stages of diabetes, it can be added in subjects who fail on multiple oral agent therapy or in insulin-treated individuals who do not reach the target glycemic control.

Progressive $\beta$-cell failure is the principle factor responsible for the progression of T2DM. Thus, preservation of beta-cell function can be expected to produce a durable reduction in $\mathrm{HbA1c}$. Studies in experimental animals and humans have reported that lowering the plasma glucose concentration with dapagliflozin improves beta-cell function, that is, reversal of glucotoxicity. Although information about the long-term durability of dapagliflozin is lacking, some studies have reported that the decrease in HbAlc produced by dapagliflozin is maintained for up to 2 years. It should be noted that these studies were extension of previous trials, and only $\sim 70$ and $\sim 90 \%$ of subjects in these two studies [24,28], respectively, entered the second-year extension study. More data will be required before making definitive conclusions about the durability of dapagliflozin. To the extent that glucotoxicity contributes to the demise in beta-cell function in subjects with impaired glucose tolerance (IGT) or impaired fasting glucose (IFG), these drugs also may prove useful in the treatment of 'prediabetes'. Lastly, unlike most currently available antidiabetic agents, dapagliflozin promotes weight loss and reduces blood pressure which would be expected to have potential cardiovascular benefit.

\section{Acknowledgments}

RA DeFronzo has received grants from Takeda, Amylin, Bristol-Myers Squibb, Speakers Bureau, BMS, Janssen and Novo Nordisk. They have been on the advisory board for Amylin, BMS, Takeda, Janssen, Novo Nordisk and Lexicon.

\section{Bibliography}

Papers of special note have been highlighted as either of interest ( ) or of considerable interest (O) to readers.

1. Danaei G, Finucane MM, Lu Y, et al. Global Burden of Metabolic Risk Factors of Chronic Diseases Collaborating Group (Blood Glucose). National, regional, and global trends in fasting plasma glucose and diabetes prevalence since 1980: systematic analysis of health examination surveys and epidemiological studies with 370 country-years and 2.7 million participants. Lancet. 2011; 378:3140. [PubMed: 21705069]

2. DeFronzo RA. Banting lecture: from the triumvirate to the ominous octet: a new paradigm for the treatment of type 2 diabetes mellitus. Diabetes. 2009; 58:773-95. [PubMed: 19336687]

3. Rossetti L, Giaccari A, DeFronzo RA. Glucose toxicity. Diabetes Care. 1990; 13:610-30. [PubMed: 2192847]

4. The American Diabetes Association. Standards of medical care in diabetes-2007. Diabetes Care. 2007; 30(Supp I):S4-S41. [PubMed: 17192377]

5. Hoerger TJ, Segel JE, Gregg EW, et al. Is glycemic control improving in U.S. adults? Diabetes Care. 2008; 31:81-6. [PubMed: 17934153]

60. Abdul-Ghani MA, Norton L, Defronzo RA. Role of sodium-glucose cotransporter 2 (SGLT 2) inhibitors in the treatment of type 2 diabetes. Endocr Rev. 2011; 32:515-31. This is a comprehensive review that summarizes renal glucose handling in normal and diabetic individuals and the metabolic actions of SGLT2 inhibitors. [PubMed: 21606218]

7. Wright EM, Loo DD, Hirayama BA. Biology of human sodium glucose transporters. Physiol Rev. 2011; 91:733-94. [PubMed: 21527736] 
8. Farber SJ, Berger EY, Earle DP. Effect of diabetes and insulin of the maximum capacity of the renal tubules to reabsorb glucose. J Clin Invest. 1951; 30:125-9. [PubMed: 14814204]

9. Mogensen CE. Urinary albumin excretion in early and long-term juvenile diabetes. Scand J Clin Lab Invest. 1971; 28:183-93. [PubMed: 5130106]

10. Meng W, Ellsworth BA, Nirschl AA, et al. Discovery of dapagliflozin: a potent, selective renal sodium-dependent glucose cotransporter 2 (SGLT2) inhibitor for the treatment of type 2 diabetes. Med Chem. 2008; 251:1145-9.

11. Komoroski B, Vachharajani N, Feng Y, et al. Dapagliflozin, a novel, selective SGLT2 inhibitor, improved glycemic control over 2 weeks in patients with type 2 diabetes mellitus. Clin Pharm Ther. 2009; 85:513.

12. Liu JJ, Lee T, DeFronzo RA. Why Do SGLT2 inhibitors inhibit only 30-50\% of renal glucose reabsorption in humans? Diabetes. 2012; 61:2199-204. [PubMed: 22923645]

13. Abdul-ghani MA, DeFronzo RA, Norton L. A novel hypothesis to explain why SGLT2 inhibitors inhibit only $30-50 \%$ of filtered glucose load in humans? Diabetes. 2013 In press.

14. DeFronzo RA, Hompesch M, Kasichayanula S, et al. Dapagliflozin reduces renal threshold for glucose excretion in type 2 diabetes. Diabetologia. 2012; 55(Suppl):A747.

15. Kasichayanula S, Liu X, Zhang W, et al. Effect of a high-fat meal on the pharmacokinetics of dapagliflozin, a selective SGLT2 inhibitor, in healthy subjects. Diabetes Obes Metab. 2011; 13:770-3. [PubMed: 21435141]

16. Obermeier M, Yao M, Khanna A, et al. In vitro characterization and pharmacokinetics of dapagliflozin (BMS-512148), a potent sodium-glucose cotransporter type II inhibitor, in animals and humans. Drug Metab Dispos. 2010; 38:405-14. [PubMed: 19996149]

17. Kasichayanula S, Liu X, Shyu WC, et al. Lack of pharmacokinetic interaction between dapagliflozin, a novel sodium-glucose transporter 2 inhibitor, and metformin, pioglitazone, glimepiride or sitagliptin in healthy subjects. Diabetes Obes Metab. 2011; 13:47-54.

18. Ferrannini E, Ramos SJ, Tang W, et al. Dapagliflozin monotherapy in T2DM patients with inadequate glycemic control by diet and exercise: a randomized, double-blind, placebo-controlled, multicenter phase III trial. Diabetes Care. 2010; 33:2217-24. [PubMed: 20566676]

19. Bailey CJ, Iqbal N, T'joen C, et al. Dapagliflozin monotherapy in drug-naïve patients with diabetes: a randomized-controlled trial of low-dose range. Diabetes Obes Metab. 2012; 14:951-9. [PubMed: 22776824]

20. Kaku K, Inoue S, Matsuoka O, et al. Efficacy and safety of dapagliflozin as a monotherapy for type 2 diabetes mellitus in Japanese patients with inadequate glycaemic control: a phase II multicentre, randomized, double-blind, placebo-controlled trial. Diabetes Obes Metab. 2012 Epub ahead of print.

21. List JF, Woo V, Morales E, et al. Sodium-glucose cotransport inhibition with dapagliflozin in type 2 diabetes. Diabetes Care. 2009; 32:650-7. [PubMed: 19114612]

22. Henry RR, Murray AV, Marmolejo MH, et al. Dapagliflozin, metformin XR, or both: initial pharmacotherapy for type 2 diabetes, a randomised controlled trial. Int J Clin Pract. 2012; 66:44656. [PubMed: 22413962]

23. Bailey CJ, Gross JL, Pieters A, et al. Effect of dapagliflozin in patients with type 2 diabetes who have inadequate glycaemic control with metformin: a randomised, double-blind, placebocontrolled trial. Lancet. 2010; 375:2223-33. [PubMed: 20609968]

24. Baily CJ, Gross JI, Yadav M, et al. Sustained efficacy of dapagliflozin when added to metformin in type 2 diabetes inadequately controlled by metformin monotherapy. Diabetologia. 2011; 54(Suppl 1):A146.

25. Nauck MA, Del Prato S, Meier JJ, et al. Dapagliflozin versus glipizide as add-on therapy in patients with type 2 diabetes who have inadequate glycemic control with metformin: a randomized, 52-week, double-blind, active-controlled noninferiority trial. Diabetes Care. 2011; 34:2015-22. [PubMed: 21816980]

26. Del Prato S, Nauck M, Rohwedder K, et al. Long term efficacy and safety of ass-on dapagliflozin vs add-on glipizide in patients with type 2 diabetes mellitus inadequately controlled with metformin: 2 year results. Diabetologia. 2011; 54(Suppl1):A852. 
27. Strojek K, Yoon KH, Hruba V, et al. Effect of dapagliflozin in patients with type 2 diabetes who have inadequate glycaemic control with glimepiride: a randomized, 24-week, double-blind, placebo-controlled trial. Diabetes Obes Metab. 2011; 13:928-38. [PubMed: 21672123]

28. Rosenstock J, Vico M, Wei L, et al. Effects of dapagliflozin, an SGLT2 inhibitor, on HbA(1c), body weight, and hypoglycemia risk in patients with type 2 diabetes inadequately controlled on pioglitazone monotherapy. Diabetes Care. 2012; 35:1473-8. [PubMed: 22446170]

29. Wilding JPH, Norwood P, Tjoen C, et al. A study of dapagliflozin in patients with type 2 diabetes receiving high doses of insulin plus insulin sensitizers. Applicability of a novel insulinindependent treatment. Diabetes Care. 2009; 32:1656-62. [PubMed: 19528367]

30. Wilding JPH, Woo V, Soler NG, et al. Long-term efficacy of dapagliflozin in patients with type 2 diabetes mellitus receiving high doses of insulin: a randomized trial. Ann Inter Med. 2012; 156:405-15.

3100. Zhang L, Feng Y, List J, et al. Dapagliflozin treatment in patients with different stages of type 2 diabetes mellitus: effects on glycaemic control and body weight. Diab Obesity Metab. 2010; 12:510-16. A nice study that demonstrates the efficacy of dapagliflozin in subjects with newonset as well as in subjects with long-standing diabetes.

32. Kasichayanula S, Liu X, Benito MP, et al. The influence of kidney function on dapagliflozin exposure, metabolism, and efficacy in healthy subjects and in patients with type 2 diabetes mellitus. Br J Clin Pharmacol. 2012 [Epub ahead of print].

33. Kohan DE, Fioretto P, List J, Tang W. Efficacy and safety of dapagliflozin in patients with type 2 diabetes and moderate renal impairment. J Am Soc Nephrol. 2011; 22:232A-3A.

34. Bolinder J, Ljunggren Ö, Kullberg J, et al. Effects of dapagliflozin on body weight, total fat mass, and regional adipose tissue distribution in patients with type 2 diabetes mellitus with inadequate glycemic control on metformin. J Clin Endocrinol Metab. 2012; 97:1020-31. [PubMed: 22238392]

35. Vallon V, Richter K, Blantz RC, et al. Glomerular hyperfiltration in experimental diabetes mellitus: potential role of tubular reabsorption. J Am Soc Nephrol. 1999; 10:2569-76. [PubMed: 10589696]

36. Thomson SC, Vallon V, Blantz RC. Kidney function in early diabetes: the tubular hypothesis of glomerular filtration. Am J Physiol. 2004; 286:F8-F18.

37. Thomson SC, Rieg T, Miracle C, et al. Acute and chronic effects of SGLT2 blockade on glomerular and tubular function in the early diabetic rat. Am J Physiol Regul Integr Comp Physiol. 2012; 302:R75-83. [PubMed: 21940401]

38. The Diabetes Control and Complications Trial Research Group. the effect of intensive treatment of diabetes on the development and progression of long-term complications in insulin-dependent diabetes mellitus. N Engl J Med. 1993; 329:977-86. [PubMed: 8366922]

39. UK Prospective Diabetes Study (UKPDS) Group. Intensive blood-glucose control with sulphonylureas or insulin compared with conventional treatment and risk of complications in patients with type 2 diabetes (UKPDS 33). Lancet. 1998; 352:837-53. [PubMed: 9742976]

40. Noonan WT, Shapiro VM, Banks RO. Renal glucose reabsorption during hypertonic glucose infusion in female streptozotocin-induced diabetic rats. Life Sci. 2001; 68:2967-77. [PubMed: 11411796]

41. Dominguez JH, Camp K, Maianu L, et al. Molecular adaptations of GLUT1 and GLUT2 in renal proximal tubules of diabetic rats. Am J Physiol. 1994; 266:F283-90. [PubMed: 8141329]

42. Nelson RG, Bennett PH, Beck GJ, et al. Development and progression of renal disease in Pima Indians with non-insulin-dependent diabetes mellitus. Diabetic Renal Disease Study Group. N Engl J Med. 1996; 335:1636-42. [PubMed: 8929360]

43. Tuttle KR, Bruton JL, Perusek MC, et al. Effect of strict glycemic control on renal hemodynamic response to amino acids and renal enlargement in insulin-dependent diabetes mellitus. N Engl J Med. 1991; 324:1626-32. [PubMed: 2030719]

44. Parikh S, Johnsson K, Ptaszynska A, et al. Characterization of urinary tract infection in the setting of pharmacologically induced glucosuria. Diabetologia. 2011; 54(Suppl):A841.

45. Ljunggren Ö, Bolinder J, Johansson L, et al. Dapagliflozin has no effect on markers of bone formation and resorption or bone mineral density in patients with inadequately controlled type 2 diabetes mellitus on metformin. Diabetes Obes Metab. 2012; 14:990-9. [PubMed: 22651373] 


\section{Box 1}

\section{Drug summary}

\begin{tabular}{|ll|}
\hline Drug name & Dapagliflozin \\
Phase & Approved for clinical use in Europe \\
Indication & Type 2 diabetes \\
Pharmacology description & Specific inhibitor of sodium-glucose cotransporter 2 \\
Route of administration & $\mathrm{PO}$ \\
Chemical structure &
\end{tabular}

Pharmaprojects - copyright to Citeline Drug Intelligence (an Informa business). Readers are referred to Pipeline (http://informa-pipeline.citeline.com) and Citeline (http://informa.citeline.com). 


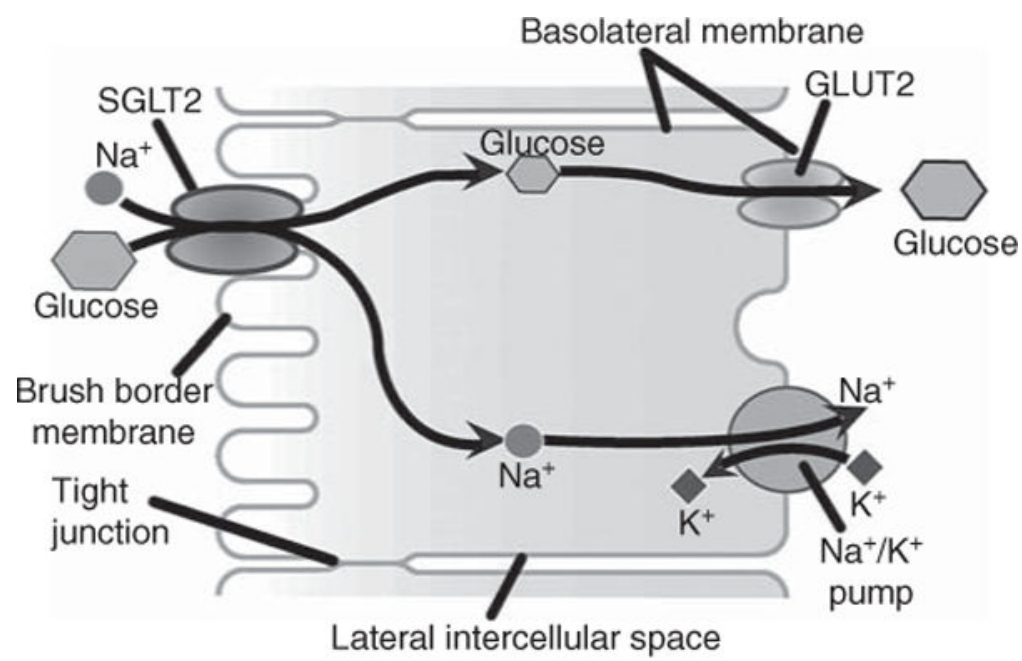

Figure 1.

A scheme of sodium-glucose uptake in pi-oxinial renal epithelium cell. 


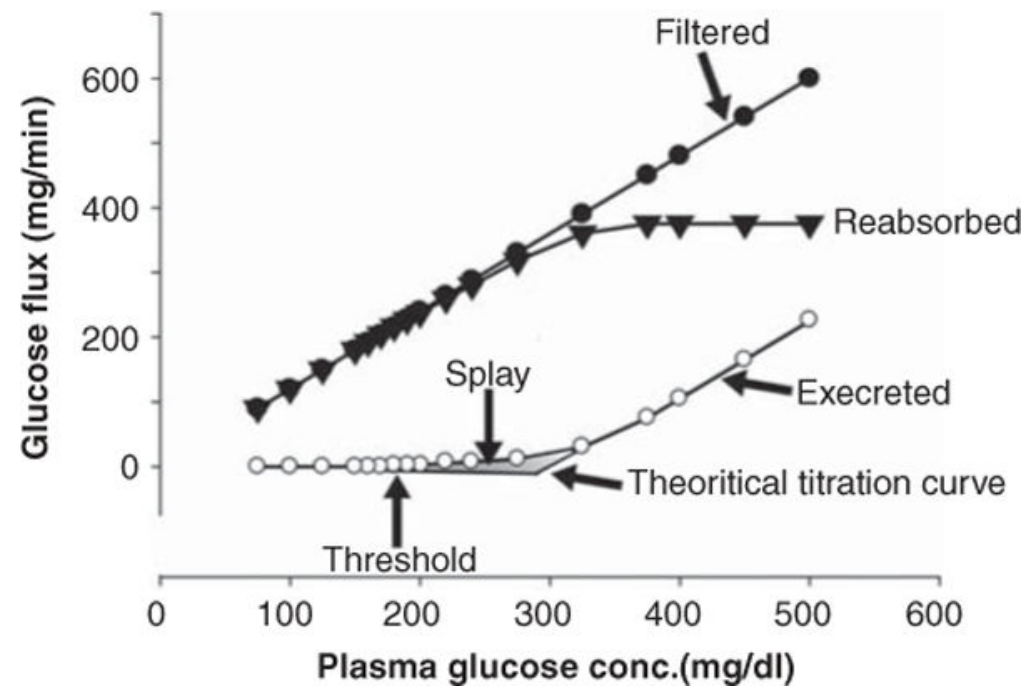

Figure 2. Kinetics of renal handling of glucose. The shaded area represents the splay Reprinted with permission from [6]. 


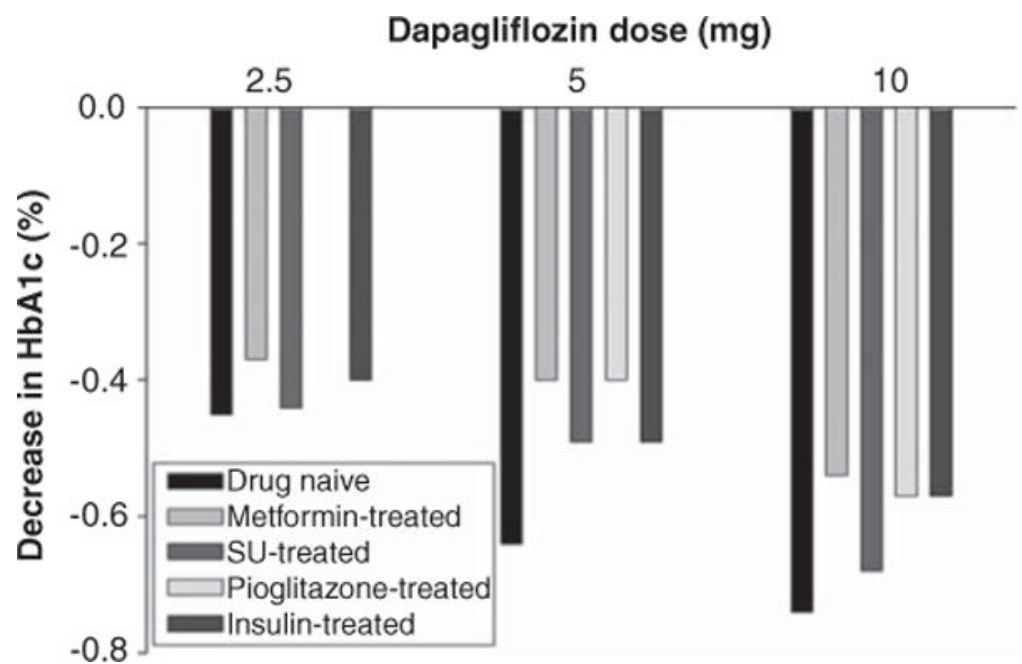

Figure 3. Efficacy of dapagliflozin in lowering the HbA1c in T2DM individuals Adapted from [15,17-20,22,24,27,39]. 


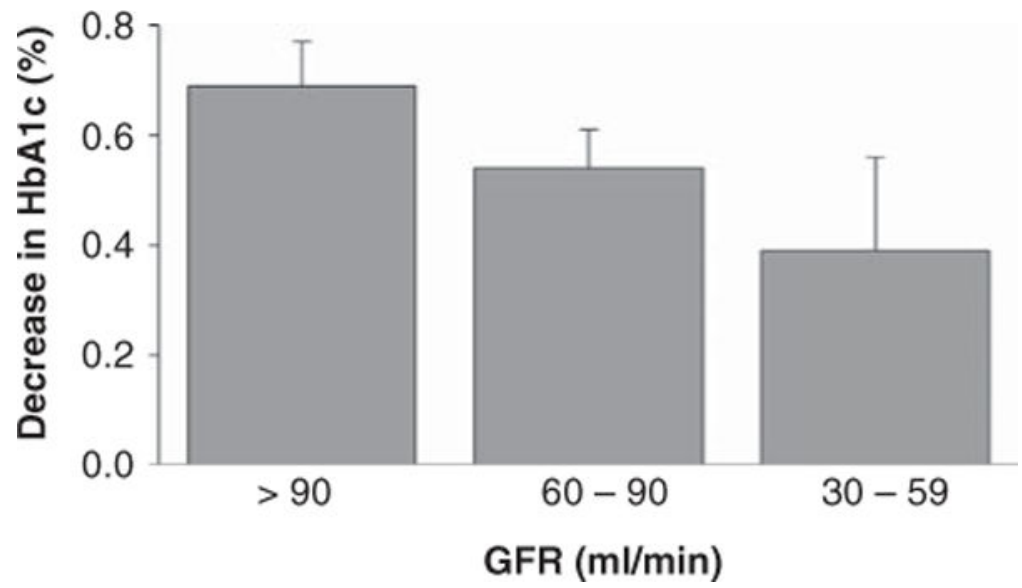

Figure 4. Impact of reduced renal function on the glucose-lowering efficiency of dapagliflozin Adapted from [29]. 